\title{
Design, Build, and Testing of TacSat Thin Film Solar Arrays
}

\author{
J William Zuckermandel, Scott Enger, and Neeraj Gupta \\ MicroSat Systems, Inc \\ 8130 Shaffer Parkway, Littleton, CO, 80126; (303) 285-1835 \\ bzuckermandel@microsatsystems.com, senger@microsatsystems.com,ngupta@microsatsystems.com
}

\begin{abstract}
MicroSat Systems, Inc. (MSI) has developed a low cost, lightweight, solar array system using thinfilm photovoltaic (TFPV) material to meet power generation needs for future responsive space missions. The Fold Integrated Thin Film Stiffener (FITS) is the deployment portion of the system. FITS is an integrated, passively deployed solar array structure designed specifically for TFPV, however a variety of photovoltaic (PV) options can be utilized by using the FITS deployment technology. FITS extends the boundaries of space PV systems by eliminating conventional rigid structures and mechanisms to maximize the lightweight and low stowage volume advantages of TFPV. FITS uses multifunctional, foldable components that store energy to provide deployment force and deployed stiffness, and have integrated power cabling to meet the demanding mass, cost and power requirements of programs like the TacSat series and anticipated future responsive space missions. MSI has completed the build and qualification test program for a two wing experimental solar array for the Air Force Research Laboratory (AFRL) TacSat-2 mission scheduled for launch in November of 2006. The array utilizes amorphous silicon (a-Si) thin-film photovoltaics on a 1-mil stainless steel substrate from United Solar Ovonic (USOC), integrated with MSI's patented FITS solar array deployment system. The experimental solar array will provide $120 \mathrm{~W}$ of additional power to the spacecraft on top of the primary solar arrays while providing valuable onorbit performance data of the TFPV to the aerospace community for future mission planning. This characterization will be done by monitoring current, voltage, and temperature of the array wings over time using an I-V electronics box built by Lockheed Martin in Littleton, CO. Currently, MSI is under contract with AFRL to design, fabricate, and test a $380 \mathrm{~W}$ EOL FITS wing while focusing on the scalability and modularity of the FITS design. For the current program, MSI is designing a FITS wing consisting of four modular strings using USOC a-Si TFPV material on polymer substrate of approximately $95 \mathrm{~W}$ EOL each. This wing design will result in a 2 wing FITS solar array of $760 \mathrm{~W}$ EOL, however, because of the module array design; another string could easily be added to each wing resulting in a $950 \mathrm{~W}$ EOL array. This paper will discuss the current status of the design, build, and test of the TacSat-2 experimental FITS solar arrays and how the lessons learned from that program are being applied to the 380 W EOL FITS solar array design. It will outline the current status of the $380 \mathrm{~W}$ EOL FITS solar array as well as the benefits of the FITS solar array technology compared to state of the art conventional rigid arrays.
\end{abstract}

\section{NOMENCLATURE}

$\begin{array}{ll}A F R L & =\text { Air Force Research Laboratory } \\ a-S i & =\text { amorphous silicon } \\ C T D & =\text { Composite Technologies Development } \\ E M & =\text { engineering model } \\ E M C & =\text { elastic memory composite } \\ E O L & =\text { end of life } \\ F I T S & =\text { fold integrated thin-film stiffener } \\ H z & =\text { hertz } \\ I_{s c} & =\text { short circuit current } \\ I V & =\text { current/voltage } \\ k W & =\text { kilowatt } \\ L E O & =\text { low earth orbit } \\ M S I & =\text { MicroSat Systems, Inc } \\ O R S & =\text { operationally responsive space } \\ P^{\max } & =\text { maximum power point } \\ P V & =\text { photovoltaic } \\ R^{2} & =\text { coefficient of determination } \\ S O A & =\text { state-of-the-art }\end{array}$

$T F P V=$ thin-film photovoltaic

$U S O C=$ United Solar Ovonic Corporation

$V_{o c} \quad=$ open circuit voltage

$W \quad=$ watt

\section{NEED AND OPPORTUNITY}

The threats to the United States are shifting from the well defined global super powers to small, rogue nations, difficult to identify and characterize. Military operations are trending toward quick strike, short duration campaigns lasting less than six months. This trend drives the requirement for space based intelligence, surveillance, and reconnaissance (ISR) systems that are agile, able to support deployment around the globe, and operational within seven days after call-up as seen in Fig. 1. Key to this paradigm is enabling small, agile, launch platforms capable of payload integration and launch in a matter of hours, and able to satisfy the full range of inclinations in a low 
earth orbit (LEO). This can only be accomplished by driving down the mass and volume of the spacecraft/payload system without sacrificing on-orbit performance.

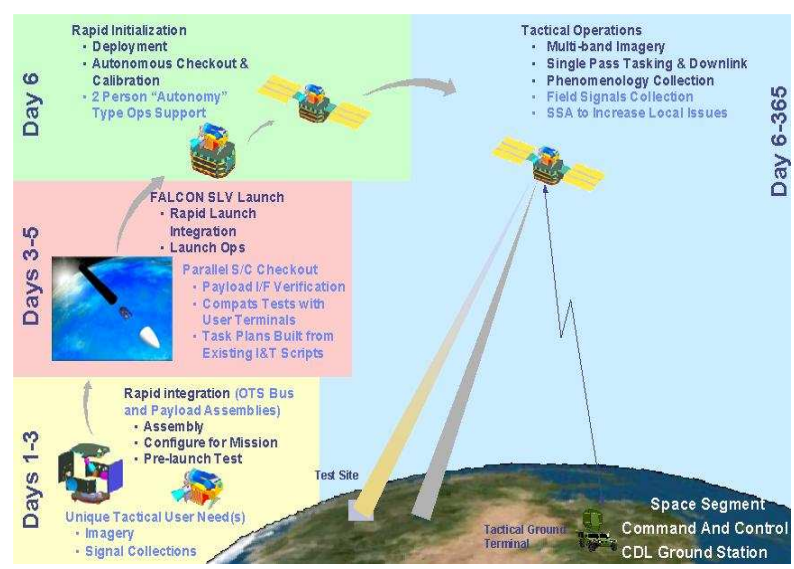

Figure 1. Responsive Space Vision

The Air Force Research Lab (AFRL) and other agencies within the United States military are interested in developing a new paradigm for spacecraft integration and test that employs a next generation, "standard" architecture. These agencies are currently undertaking an initiative that seeks to provide operationally responsive access and near term tactical exploitation of space.

TacSat- 1 and TacSat- 2 are the first experiments in this initiative to exploit access to space within the operational contingency planning cycle. The broader system characteristic toward responsive space constitutes an operationally relevant space capability that takes advantage of a highly automated microsatellite bus, modular payloads, standard interfaces, tasking and data dissemination using standard protocols, and low cost rapid-response launches. Each experiment tests key elements needed for a deployable Operationally Responsive Space (ORS) system.

In order to provide rapid and affordable spacecraft for ORS platforms, the creation of a standardized spacecraft bus has been identified as necessary by many organizations within the military. As well, spacecraft subsystems must become modular and standardized to support the aggressive schedules and cost of most ORS missions. One subsystem that does not support the ORS paradigm is the power subsystem, specifically the solar arrays. A substitute is needed for conventional rigid solar arrays that do not have the specific power required to meet the ever increasing needs for the surveillance and tracking sensors proposed for spacebased missile defense. Conventional arrays are also typically long lead and extremely cost prohibitive for more responsive missions. MSI's thin-film photovoltaic solar arrays provide a lightweight, low cost solution for power generation for future experimental and operational space-based platforms.

\section{INNOVATIVE SOLUTION}

MicroSat Systems, Inc. has completed the development of a patented solar array deployment system called "FITS",. The FITS uses thin-film photovoltaic material to create a low-cost, high specific power, and highly efficient stowage/deployment system that is capable of meeting the requirements of ORS spacecraft.

FITS is an integrated, passively deployed, solar array

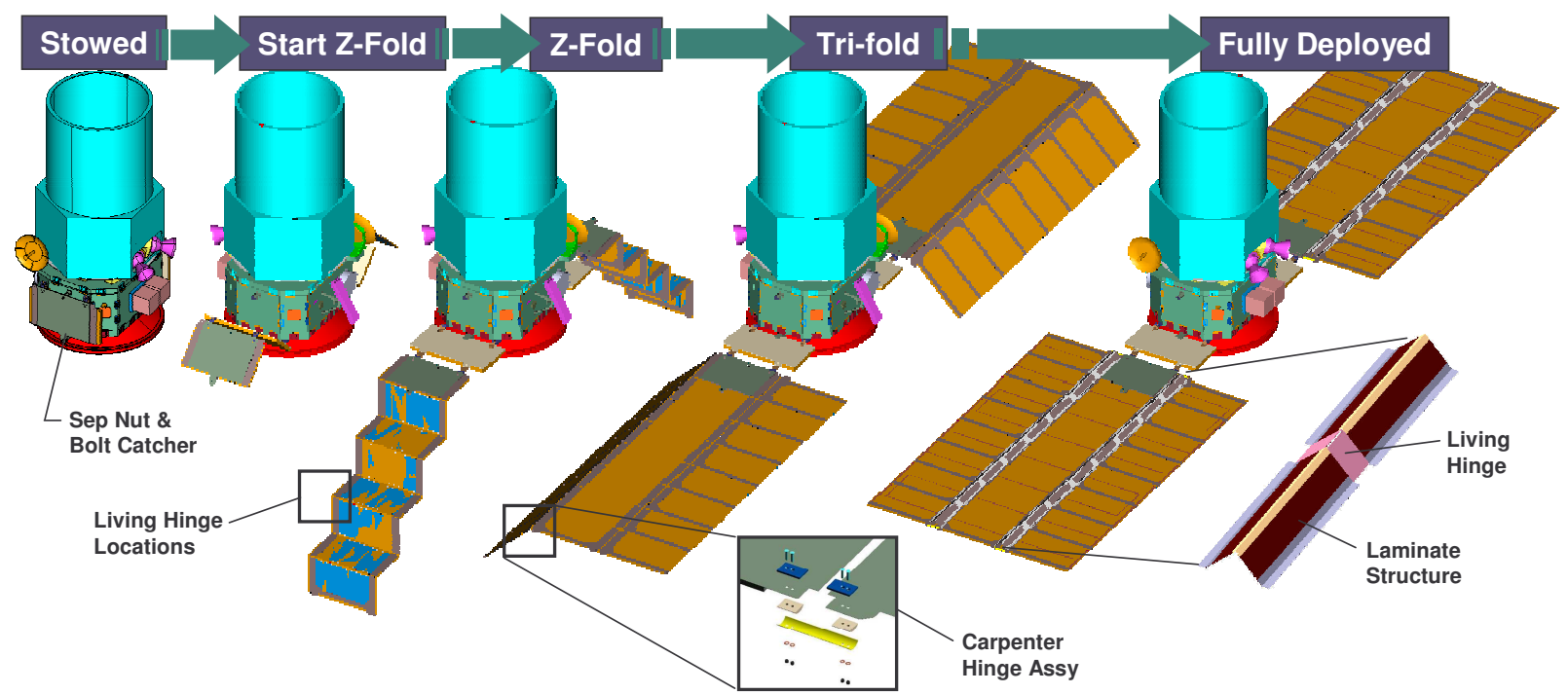

Figure 2. FITS Deployment Sequence Shown On MSI Bus 
structure designed specifically for TFPV arrays. FITS technology extends the applications of state-of-the-art (SOA) space PV systems by eliminating conventional rigid structures and mechanisms in order to maximize the lightweight and low stowage volume advantages of TFPV. FITS uses multifunctional, foldable components that store energy to provide deployment force and deployed stiffness, and has integrated power cabling to meet the demanding mass and volume requirements of the TacSat programs and anticipated future missions. The simplicity of the mechanisms in the FITS deployment scheme reduces many of the failure modes associated with traditional rigid solar arrays. Each component and mechanism in the FITS system is designed to have multiple functions to eliminate mass.

FITS utilizes deployed-depth stiffening members that allow for compact stowage and unassisted deployment using a simple tri-fold and $\mathrm{z}$-fold scheme. The $\mathrm{z}$-folded joints have stored potential energy that provide deployment force using a polymer-based living hinge developed by MSI. The tri-fold joints complete the FITS deployment using force from carpenter tape hinges integrated into the base structure of the wing that lock the FITS stiffeners open once deployed. The completed deployment offers sufficient array stiffness to meet deployed natural frequencies above $0.5 \mathrm{~Hz}$. The deployment sequence of a typical FITS solar array can be seen in Fig. 2.

Using TFPV efficiencies available today, MSI's FITS system can provide a fifty percent mass, cost, and stowed volume reduction over that of conventional rigid arrays as seen by Fig. 3. These reductions are critical for meeting ORS needs because it allows the customer to fly a larger payload with a highly capable spacecraft bus on a smaller more responsive launch vehicle.

\section{TACSAT-2 EXPERIMENTAL SOLAR ARRAY}

The first goal of this Phase II program was to design, fabricate, and perform protoflight level testing of a two wing FITS TFPV solar array. This experimental solar array will fly on the AFRL TacSat-2 mission in November of 2006. The baseline design for the TacSat2 experimental solar array consisted of two FITS solar array wings using thin-film photovoltaics on a 1-mil stainless steel substrate provided by United Solar Ovonic Corporation (USOC). Each of these wings is integrated to the end of the main rigid solar array wings of the spacecraft using a restraint system as seen in Fig. 4. One wing uses a traditional torsion spring hinge while the other wing uses elastic memory composite (EMC) hinges provided by Composite Technology Development (CTD). The second objective is to gather vital flight data to validate the predicted performance of the TFPV. This flight data is collected using an IV electronics box designed and built by Lockheed Martin.

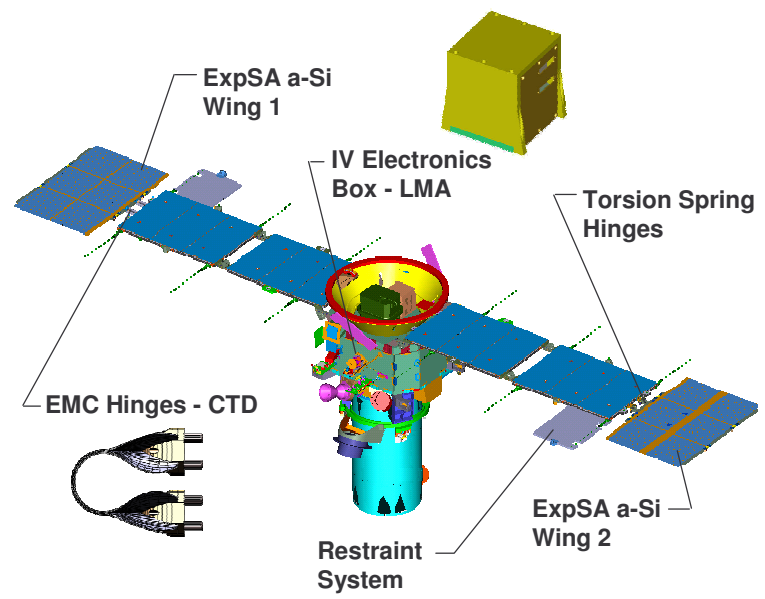

Figure 4. TacSat-2 Experimental Solar Array Payload

\begin{tabular}{|c|c|c|c|}
\hline PARAMETRIC & SOA & FITS TODAY & DESIGN GOAL \\
\hline$\overline{\text { Specific Power (W/kg) }}$ & $5 \overline{0 \text { to } 106}$ & 150 & $>500$ \\
\hline Stowed Volumetric Power (kW/m3) & 8 to 25 & 40 & $>75$ \\
\hline Array System Cost $(\$ / W)$ & $2500+$ & 1500 & $<500$ \\
\hline
\end{tabular}

Figure 3. FITS Solar Array Metrics vs. State-of-the-Art Conventional Solar Arrays

The obvious benefits of the FITS solar array deployment system led to a Phase I STTR program. The objective of this Phase I was to further the technology and prove the feasibility of its use in space. The work completed on the Phase I led to a follow-on Phase II with the objective of providing a FITS solar array flight demonstration for the AFRL TacSat-2 mission.
During IV Acquisition Mode, the FITS solar array string switches are set to open, removing the experimental solar array wings from the bus. The IV electronics will characterize each wing separately, acquiring five sets of curve data for each. The IV characterization for each wing will be conducted using eight analog current increments of increasing magnitude applied to a programmable electronic load. These proportional array current increments form a feedback loop ensuring accurate current regulation 
while measuring array voltage. This data will then be stowed in a buffer on the spacecraft until it can be downlinked to the ground.

\section{Design}

The design of the FITS experimental solar array is unique because it is attached to the outer ends of the main solar arrays of the spacecraft. MSI worked with the TacSat-2 program to identify all of the driving requirements for the payload and determined the best orientation to fly the FITS arrays and deploy the restraint system. The design of the arrays consisted of the TFPV blanket assembly, composite base structure assembly, FITS stiffener assembly, tri-fold hinge assembly, electrical assembly, and the restraint system assembly, as seen in Fig. 5.

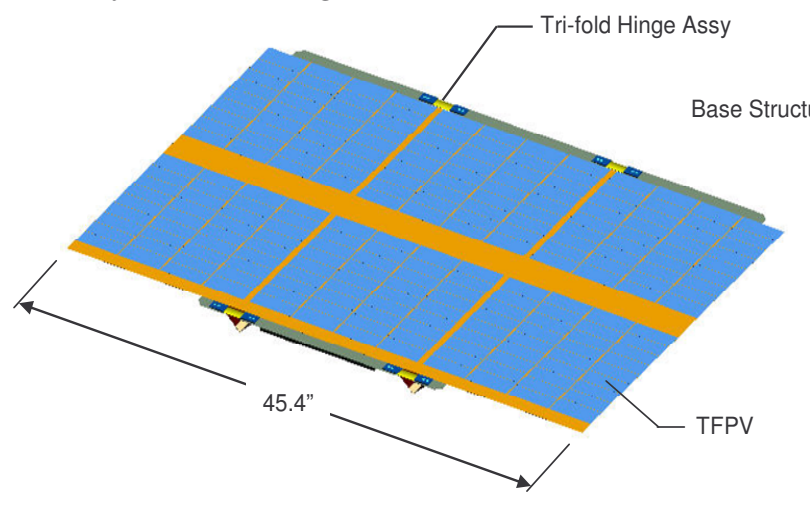

survival through the launch environment. Once the TacSat-2 spacecraft is separated from the launch vehicle and the main arrays deploy, the FITS separation nuts are fired, the restraint panel opens and the z-fold portion of the deployment begins, followed by the trifold, resulting in a fully deployed FITS solar array.

\section{Fabrication}

Upon completion of the baseline design for the experimental solar array, MSI built an engineering model of one TacSat-2 experimental solar array wings. This model was used to validate fabrication processes and integration techniques using specialized tooling designed for cell integration. Upon a successful validation of all manufacturing processes, MSI began fabrication of the detailed array components for the

Figure 5. 60W EOL Experimental Solar Array Blanket Design, a) TFPV side, b) Structure Side

The TFPV blanket assembly consists of the active cell material, interconnects, bypass diodes, substrate, backwiring, and string terminations. The backwiring is run in a serpentine fashion, as seen in Figs. 5 and 7, to cancel any current loops that can adversely affect the spacecraft attitude control system. The base structure assembly is made of multiple thin composite laminates that are designed to provide stiffness to the array when deployed but also protect its vital critical components by providing a load path to the root deployment hinges. The FITS stiffeners interface with the base structure, provide the $\mathrm{z}$-fold deployment force to the array and provide deployed-depth to create the required stiffness once deployed. The tri-fold hinge assembly provides the deployment force to the tri-fold wings once the $\mathrm{z}$ fold portion of the deployment is complete. The tri-fold hinges also provide a locking mechanism that keeps the wings fully open once deployed. The electrical assembly connects all of the strings and telemetry sensors to the bulkhead connector that interfaces with the spacecraft bus. Finally, the restraint system interfaces the FITS wing with the main solar array. It also maintains an even preload on the assembly for flight wings. (Fig. 6).

MSI then began the blanket integration of the arrays. First, the module alignment tooling was utilized to bond the modules onto the blanket substrate and interconnect

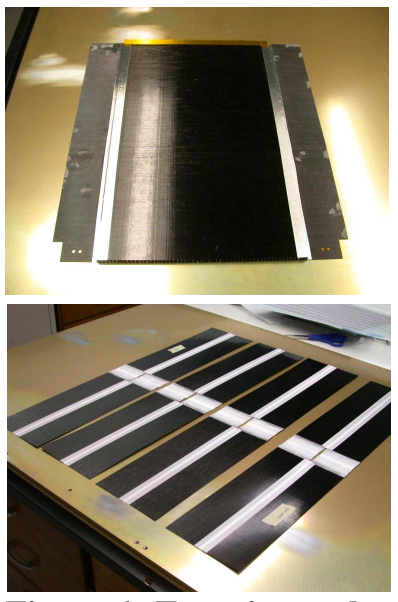

Figure 6. Experimental
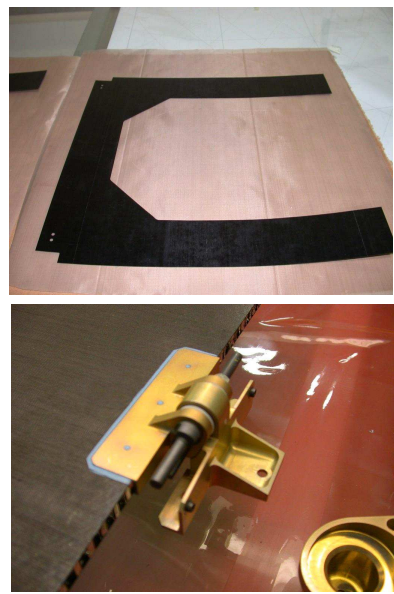

Solar
Array
Detail Structure Laminate, c) FITS Stiffener Sections, d) Deployment Hinge Assembly 
modules into strings on the array wing. The modules are then interconnected using electrical interconnects per an MSI developed process. Next, the TFPV blanket is turned over and the backwiring is integrated using an electrically conductive material encapsulated in a Kapton sleeve, which isolates it from both the ESD surface on the backside of the blanket substrate and the atmosphere. The backwiring traces the current path from the front side of the blanket eliminating the current loops. The next process is the addition of the base structure to the blanket. This is the thin laminate structure that aids in the stiffening and deployment of the FITS array wing. Finally, the FITS stiffeners are integrated onto the array wing. The buildup of these processes can be seen in Fig. 7 below.

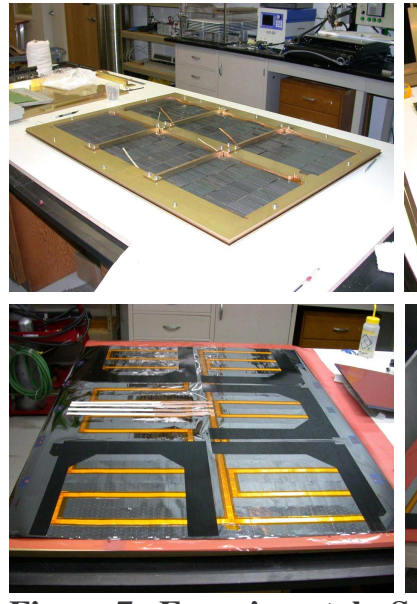

Figure 7. Experimental Solar Array Fabrication Pictures, a) Alignment Tooling \& TFPV, b) Backwiring Integration, c) Base Structure Integration, d) FITS Stiffener Integration

Finally, the experimental solar array wings are integrated with a bus simulator tool as seen in Fig. 8. This tool serves two purposes: it simulates the interfaces to the TacSat-2 spacecraft bus and it also serves as a random vibration fixture during environmental testing of the FITS array. During this time the electrical harness, blocking diodes, temperature sensors, and tri-fold hinges are also integrated onto the array wings.

\section{Protoflight Testing}

Upon completion of the fabrication of the experimental solar array wings, MSI began environmental testing. MSI decided to test each wing to protoflight levels and durations in order to fully qualify the design of the array. The testing of the array wings followed the flowchart seen in Fig. 8, which included an electrical verification of the performance after each set of
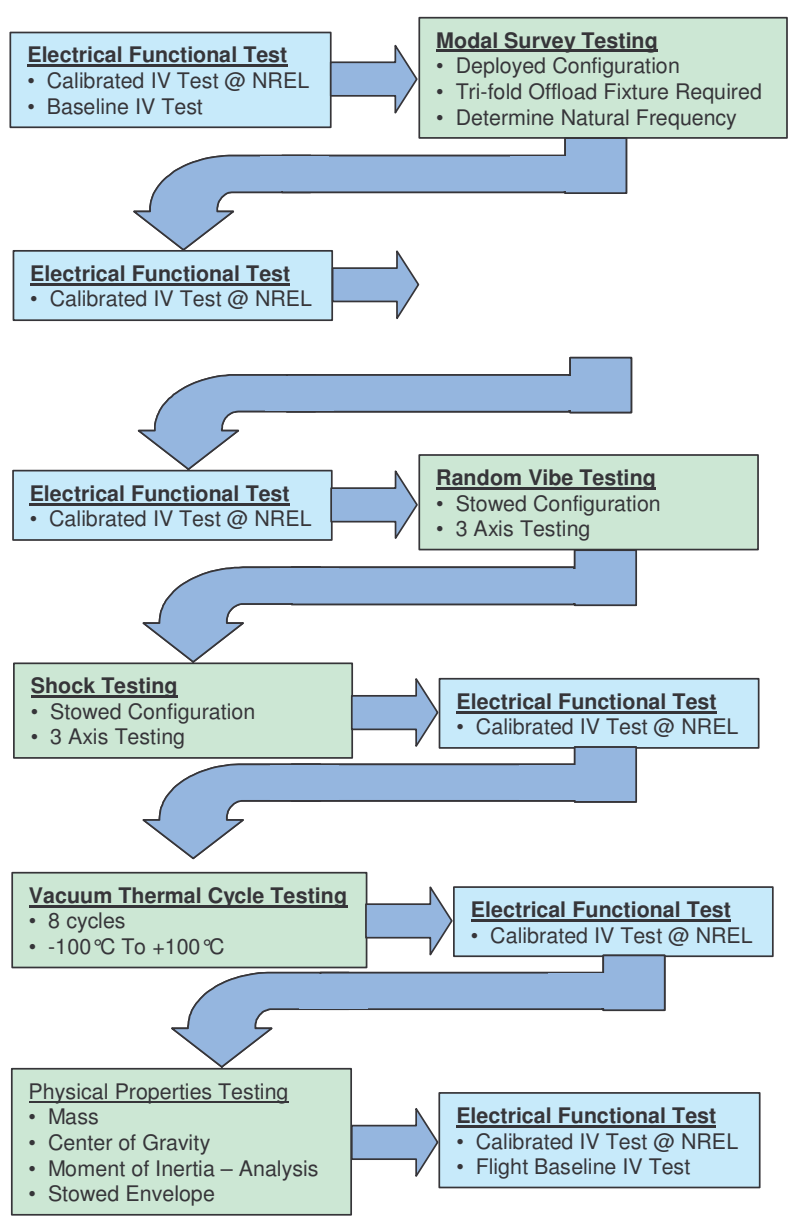

Figure 8. Experimental Solar Array Test Flow

environmental tests to verify that the testing had not damaged the array or degraded performance.

The ground deployment testing was performed at CU Boulder at the Center for Aerospace Structures, as seen in Fig. 9. The multi-axis kinematics of the FITS array deployment presented many challenges that can typically be resolved by traditional suspension system configurations. CU Boulder was subcontracted during the program to develop a kinematic model of the array deployment and validate that model with testing. The goal of this model is to analyze larger more complex FITS arrays. Videometry data was used during the deployment testing to determine the deployment angle as a function of time for each. The approach used was to isolate the three kinematic stages of the deployment and independently test them as they were modeled. The stages of the FITS deployment testing were the root hinge, z-fold, and tri-fold deployments.

The Engineering Model (EM) and flight arrays were used during deployment testing. The error associated with each stage varies widely. Many trials were conducted for the EM; however, few trials were allowed for the flight hardware in order to limit fatigue 

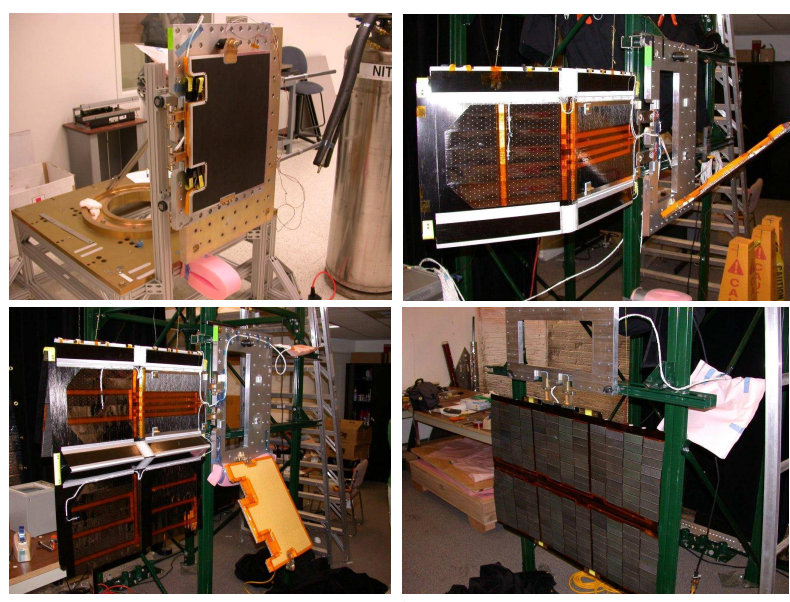

Figure 9. Experimental Solar Array Deployment Testing, a) Stowed Configuration, b) Z-fold Deployment, c) Tri-fold Partially Deployed, d) Fully Deployed Wing

loading of the flight hardware. Thus, the EM data has significantly lower uncertainties than both the flight models.

First, the z-fold and tri-fold hinges were restrained and only the root deployment hinge and interface panel were deployed. This testing occurred on the flight unit only because a full restraint system was not available for the EM. The results of this test vs. the simulation prediction can be seen in Fig. 10. The results of this test show that the simulation prediction matches the actual testing of the root deployment with a coefficient of determination $\left(\mathrm{R}^{2}\right)$ value of 0.9910 . From these results it can be determined that the assumptions made for the torsion springs and panel stiffness for the simulation are valid and can be used for future array deployment simulations.

Next, the z-fold deployment testing was performed. To do this, the tri-fold hinges were restrained and only the z-fold portion of the deployment was released. This

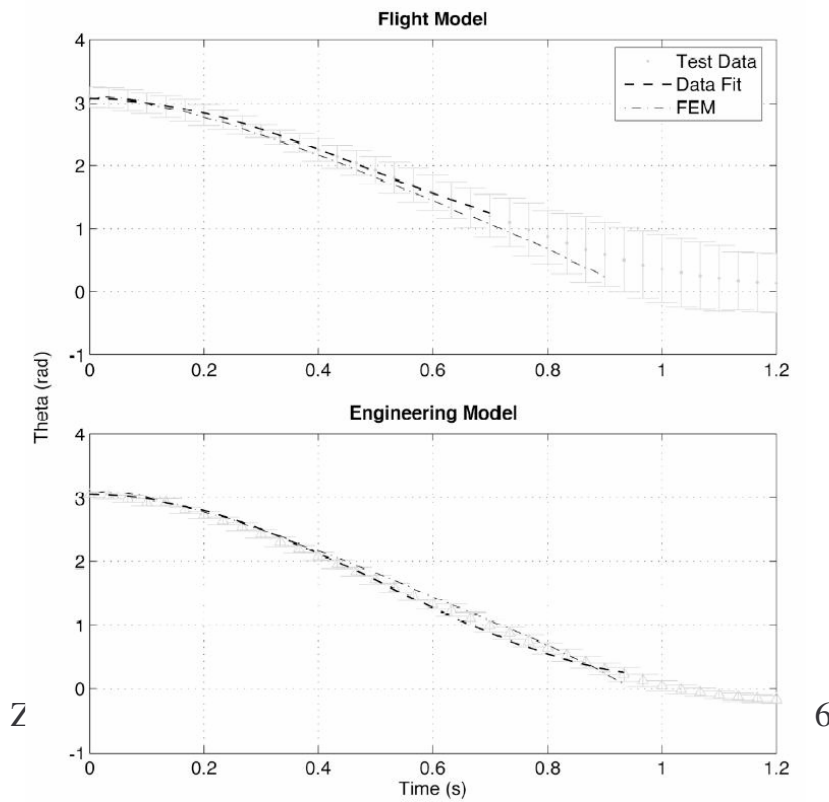

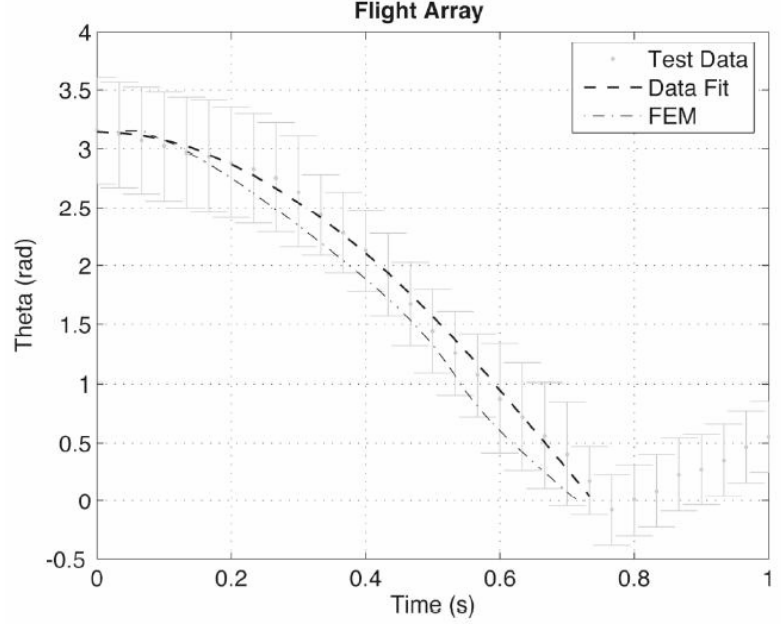

Figure 10. Root Hinge Deployment Results ${ }^{1}$

testing was performed both on the EM and on the flight unit. The results, shown in Fig. 11, show an $\mathrm{R}^{2}$ value of 0.9952 for the simulation prediction and the actual test data. This validates the simulation assumptions of the living hinge properties and blanket stiffness. From comparison of the EM and flight unit data, it can be seen that the living hinges do not have any significant degradation in performance after numerous deployments. This data also gives MSI validation that the manufacturing processes that are used to fabricate the living hinges are repeatable.

Finally, the tri-fold deployment testing was performed. This testing was performed initially on the EM unit, however, slight design changes to the flight units determined that only the flight units be used for comparison of the simulation data to the test data. During this testing, the FITS wing was rotated 90 degrees on the fixture so that the tri-fold hinges could be fully off-loaded during the deployment. The results of the flight unit tests produced an $\mathrm{R}^{2}$ value of 0.9938 and can be seen in Fig. 12. During this testing, Panel 1 was on the outside, therefore as seen in both figures, it opens prior to Panel 2. This data demonstrates that the assumptions that were made for the restoring torque and stiffness of the carpenter hinge mechanism in the simulation were accurate.

Through successive updates of the integrated computational model, the deployment of each individual stage was verified and confidence was established that the coupled deployments would be successfully simulated using these models. To verify this, test data was taken for the Root and Z-Fold combined deployment. This is the only coupled deployment test that could be performed without a complex offload fixture design or a zero-g flight, since both the Root and Z-Fold deploy in the same plane. A simulation was run for the Root and Z-Fold combined deployment with updated deployment stage parameters. The test results and the simulation results were found to

Figure 11. Z-fold Deployment Test Results ${ }^{1}$ 

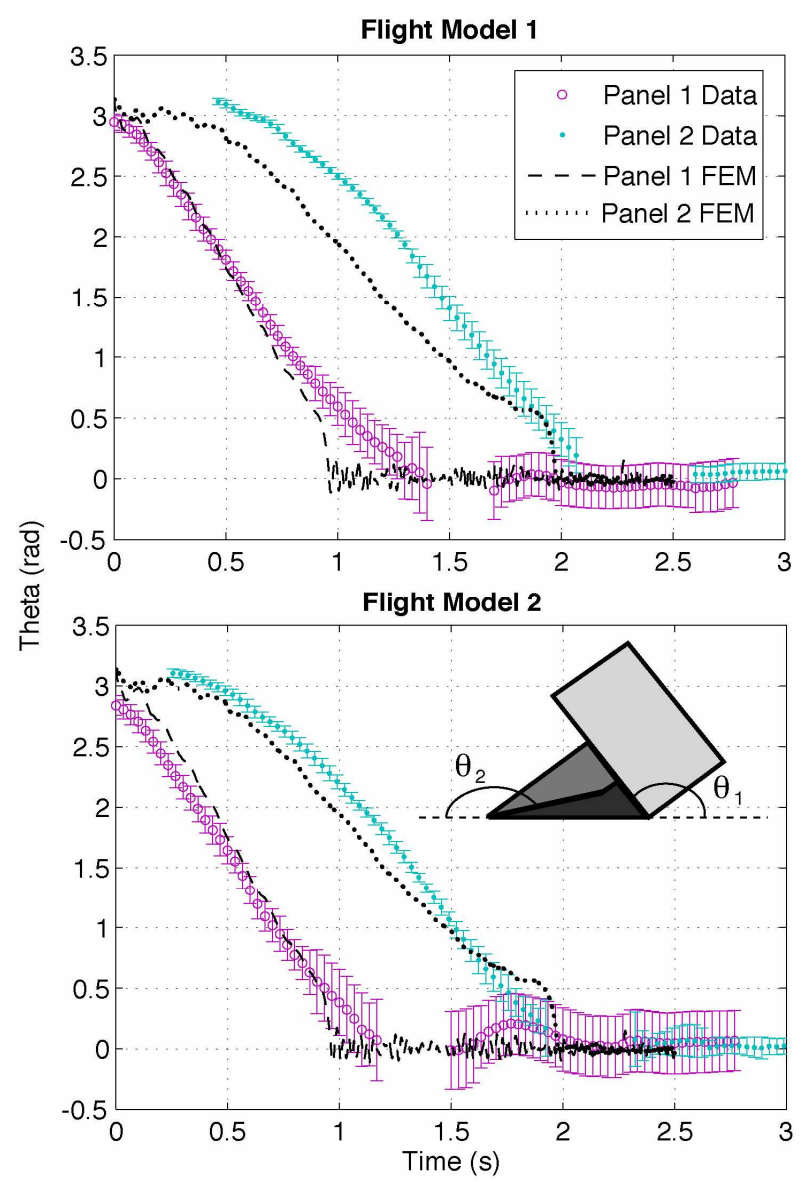

Figure 12. Tri-fold Deployment Test Results ${ }^{1}$

match each other within the measurement precision of the test which helped to validate the staged deployment testing approach as well as the coupled modeling assumptions. The test results for the root hinge and $\mathrm{z}$ fold coupled deployment can be seen in Fig. 13.

The close agreement of the combined deployment simulation with test data indicates that the methodology used has validity and warrants further investigation. By testing each individual stage of a complex deployment, and using those test results to update and refine simple single staged models, a combined multi-stage simulation of the deployment can predict the complex interactions between the stages. Implementation of this methodology, including more rigorous testing, can provide further support for this method of simulating complex deployments. In addition, on-orbit or zero-g testing of deployments and comparison to staged ground test results and combined multi-stage deployment simulations would be highly valuable.

At the completion of the ground deployment testing and post test IV characterization, MSI moved forward with the rest of the environmental testing per the test flow established in Fig. 8. The random vibration testing was performed in all three axes with the $\mathrm{X} \& \mathrm{Z}$ Axis taken to $12.46 \mathrm{G}_{\mathrm{rms}}$ and the $\mathrm{Y}$ axis taken to $14.98 \mathrm{G}_{\mathrm{rms}}$. Shock testing was performed to $1550 \mathrm{G}$ 's max. The levels for both the random vibration and shock test were established by the TacSat-2 program Environmental and Loads Document. Following these tests and their

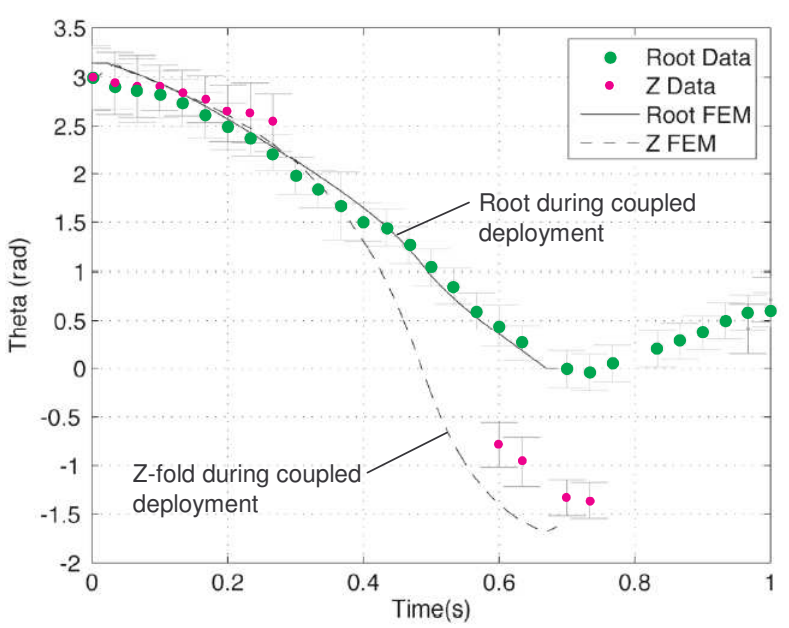

Figure 13. Root / Z-fold Coupled Deployment Results $^{1}$

subsequent IV characterization test, the array wings were exposed to thermal vacuum testing. During this test, the arrays were subjected to 8 cycles from $-100^{\circ} \mathrm{C}$ to $+100^{\circ} \mathrm{C}$ with a functional test performed at both the hot and cold dwell. This functional test ensured that the TFPV strings functioned electrically at the extreme temperatures that it will be exposed to during flight. Pictures of the environmental testing can be seen in Fig. 14 below.
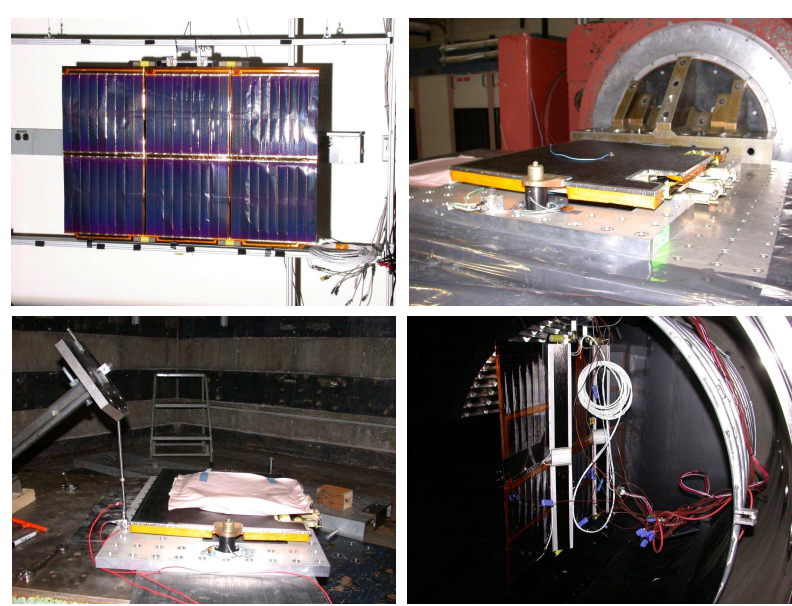

Figure 14. Experimental Solar Array Testing, a) IV Characterization, b) Random Vibration Testing, c) Shock Testing, d) Thermal Vacuum Testing

After the environmental testing was completed, MSI analyzed the test results to determine if any of the testing had degraded the array performance. IV test data was plotted and the results analyzed. It can be seen in Fig. 15 that the test results of each IV test were almost identical to the previous test. The $\mathrm{I}_{\mathrm{sc}}$ and $\mathrm{P}_{\max }$ were almost identical with slight variation of about $3 \%$ 
seen in the $\mathrm{V}_{\mathrm{oc}}$. Upon investigation it was found that this was most likely due to the test equipment measurement accuracy of $\pm 5 \%$.

\section{a-Si ExpSA AM1.5 IV Curves}

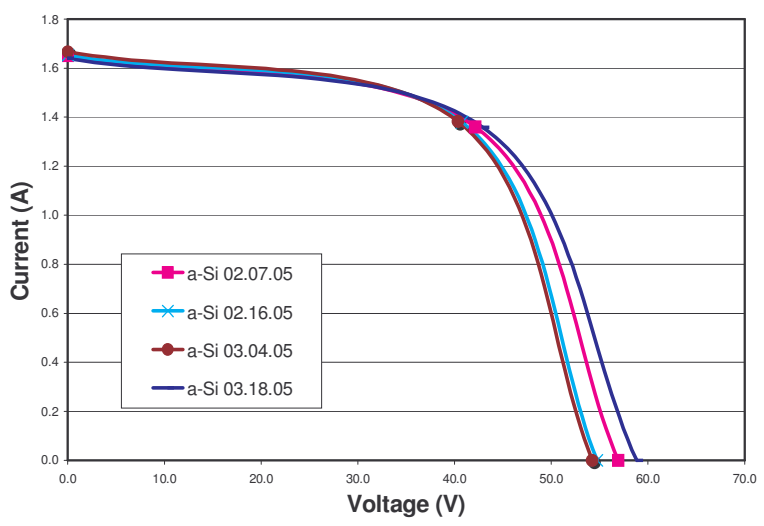

Figure 15. Experimental Solar Array IV Test Results During Environmental Testing

Since completion of the experimental solar array subsystem testing, MSI has integrated the experimental solar arrays onto the main solar arrays of TacSat-2. The main solar arrays were then integrated onto the TacSat-2 spacecraft which underwent spacecraft environmental testing in the May-June timeframe of 2006 at AFRL's Kirtland facilities. A picture of the experimental solar arrays integrated onto the TacSat-2 spacecraft during random vibration testing can be seen in Fig. 16.

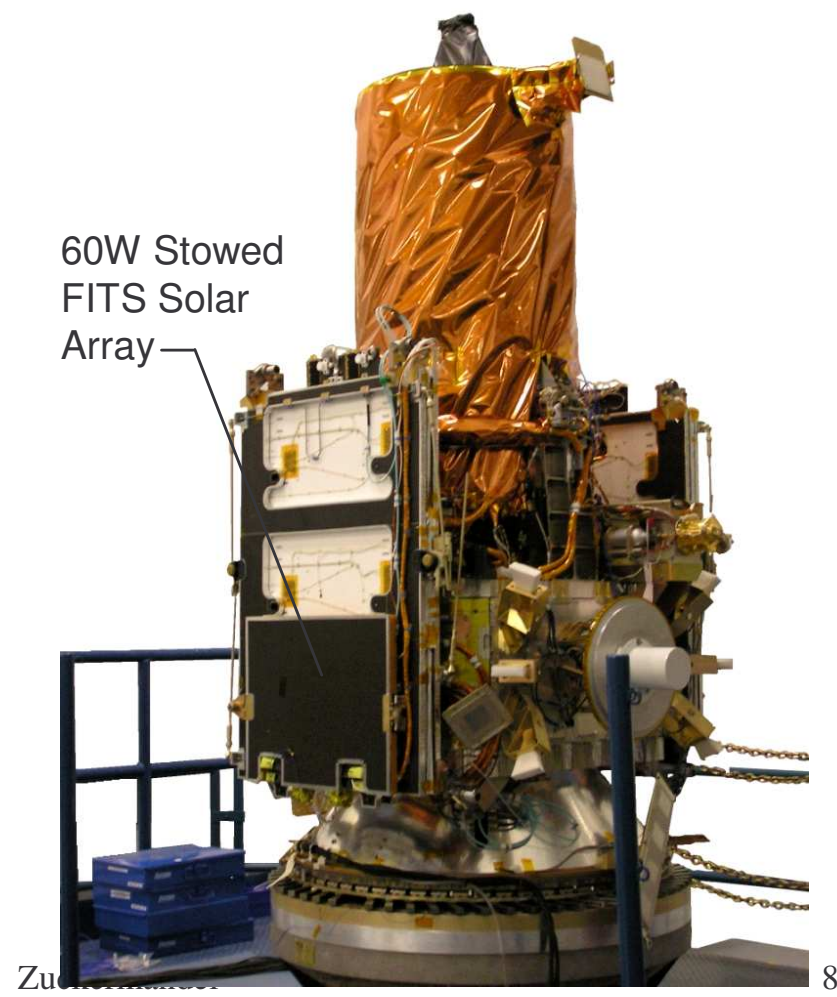

Figure 16. TacSat-2 System Vibration Testing

\section{W EOL FITS SOLAR ARRAY}

A successful launch of TacSat-2 in November of 2006, followed by deployment of the experimental solar array wings will begin to validate the work MSI has done in the development of the FITS solar array. However, development is still required on the array technology in the areas of scalability, standardization, and modularity in order to improve the FITS arrays and give them an even greater advantage over traditional rigid arrays.

After completion of the experimental solar array wings, MSI was funded by AFRL to design, build, and test a larger FITS solar array to support ORS missions such as the TacSat programs. One of the main goals of this program was to develop a solar array system that was modular and scalable in design. MSI first examined typical responsive spacecraft power requirements. After completion of this trade study, MSI determined that a modular FITS solar array system in the $1 \mathrm{~kW}$ class would meet the requirements of reposonsive spacecraft today and in the future. Although a flight qualified FITS array was designed and built for the TacSat-2 mission, there are still many technical obstacles which must be explored in order to achieve a modular design. Many of these obstacles are in the area of manufacturing and fabrication processes necessary to build a modular system.

For this program MSI has again designed the TFPV blanket using USOC thin-film photovoltaic material. However, through recent technological developments, USOC has been able to deposit this thin-film PV composition on a polymer substrate rather than their standard stainless steel substrate, thus realizing significant mass savings. This development has also eliminated the magnetics issue associated with the stainless steel product.

With these advantages came some technical hurdles as well. The first being that the polymer cells are larger cells operating at a higher current than the stainless steel substrate cells. MSI worked with USOC to develop a smaller polymer cell product that made the design of the FITS blanket a better technical solution for a modular solar array. MSI is designing each string to be compatible with a $28+/-6$ VDC bus, the standard bus voltage for most small, tactical satellites. This string will become the building block of the modular FITS solar array design. These strings could be fabricated and stored at the depot level awaiting call-up and be integrated into a flight array in one week or less. The FITS solar array blanket layout for this program can be seen in Fig. 17. The blanket is a four string design, with each string consisting of 30 series 


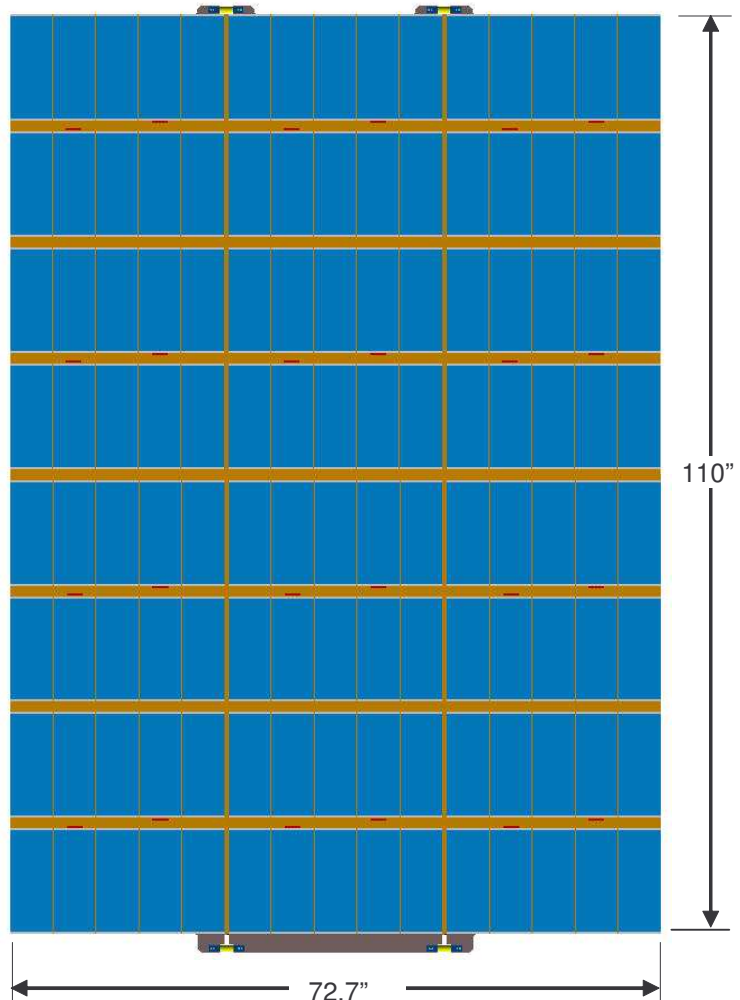

Figure 17. 380 W EOL FITS Solar Array Wing Layout

connected polymer cells. Each string has a maximum EOL voltage of $39 \mathrm{~V}$, EOL current of $2.44 \mathrm{~A}$, and EOL power of $95 \mathrm{~W}$. For this blanket configuration, the EOL max power is $380 \mathrm{~W}$, however because of the modular design, another string could be added with only a top level drawing change resulting in a $475 \mathrm{~W}$ EOL wing.

The other technical hurdle on this program was how to integrate the polymer cells. During the experimental solar array integration, tooling was designed specific to the size of the array wing being fabricated however, for this program MSI designed tooling that could be used for any size TFPV string necessary to support ORS spacecraft needs in the future. The tooling uses a large vacuum tool to lay down an entire string at one time up to string sizes of 48 " x 84 ", which provides options for future FITS solar array designs.

The restraint system of the $380 \mathrm{~W}$ EOL FITS wing is a more traditional design than the experimental solar array because it interfaces directly to the spacecraft bus. It has two separation devices which preload the array PV between two composite honeycomb panels during launch. When the separation nuts are fired, the panels deploy using traditional torsion spring driven hinges. The design of the entire $380 \mathrm{~W}$ EOL FITS wing can be seen in Fig. 18.

During the design phase of the modular FITS wing, MSI performed detailed stress analysis on the array wing components to ensure that there were sufficient margins on all components to survive launch loads, deployment loads, and on-orbit loads. MSI has completed the procurement of all parts and began fabrication of the solar array strings in May of 2006. The FITS solar array wing will be completed in July of 2006 and will subsequently begin full qualification testing. Fig. 19 shows status pictures of some of the current components of the $380 \mathrm{~W}$ EOL FITS solar array wing.

\section{MODULAR FITS DESIGN SUPPORTING ORS MISSIONS}

One of the current program goals is to develop a solar array subsystem that is capable of supporting the ORS paradigm by reducing subsystem schedule and cost. MSI is focusing on creating a standardized, modular, flexible, thin-film solar array that will be a stand alone subsystem that can be delivered to the customer in a matter of days and integrated onto the spacecraft in hours. In order to accomplish this, standard interfaces from the solar array to the bus need to be identified which includes power, mechanical, and telemetry interfaces.

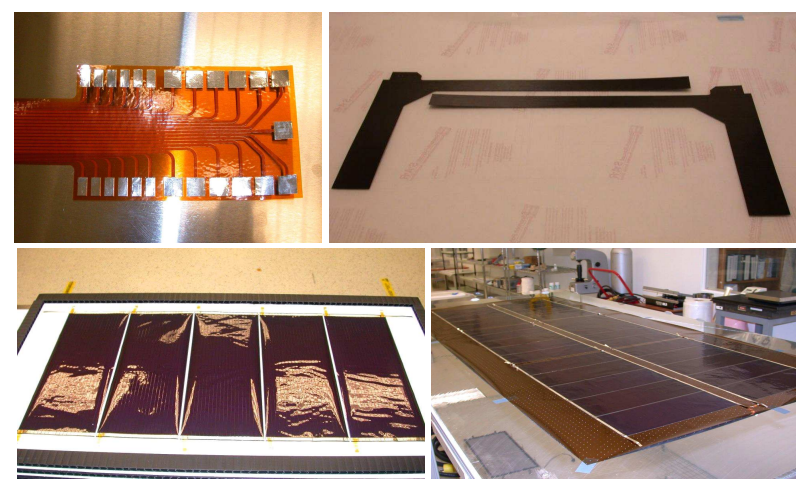

Figure 19. $380 \quad$ W $\quad$ EOL FITS Solar Array Fabrication Status, a) Flex Cable, b) Base Structure Laminates, c) a-Si Polymer Modules, d) $1 \mathrm{~kW}$ Class FITS String

During the design phase of the current contract, MSI has created a standard, modular engineering approach to the FITS solar array build that requires generation of only a top level assembly drawing for each unique array design, drastically reducing the non-recurring engineering schedule and cost. MSI has identified a scheme that would make the FITS solar array strings modular components, becoming the building block of the blanket assembly. They can be fabricated and stored at the depot level awaiting call-up. Each string would be fully electrically interconnected and have its own structural members integrated into it. As additional power is required, additional strings would be 


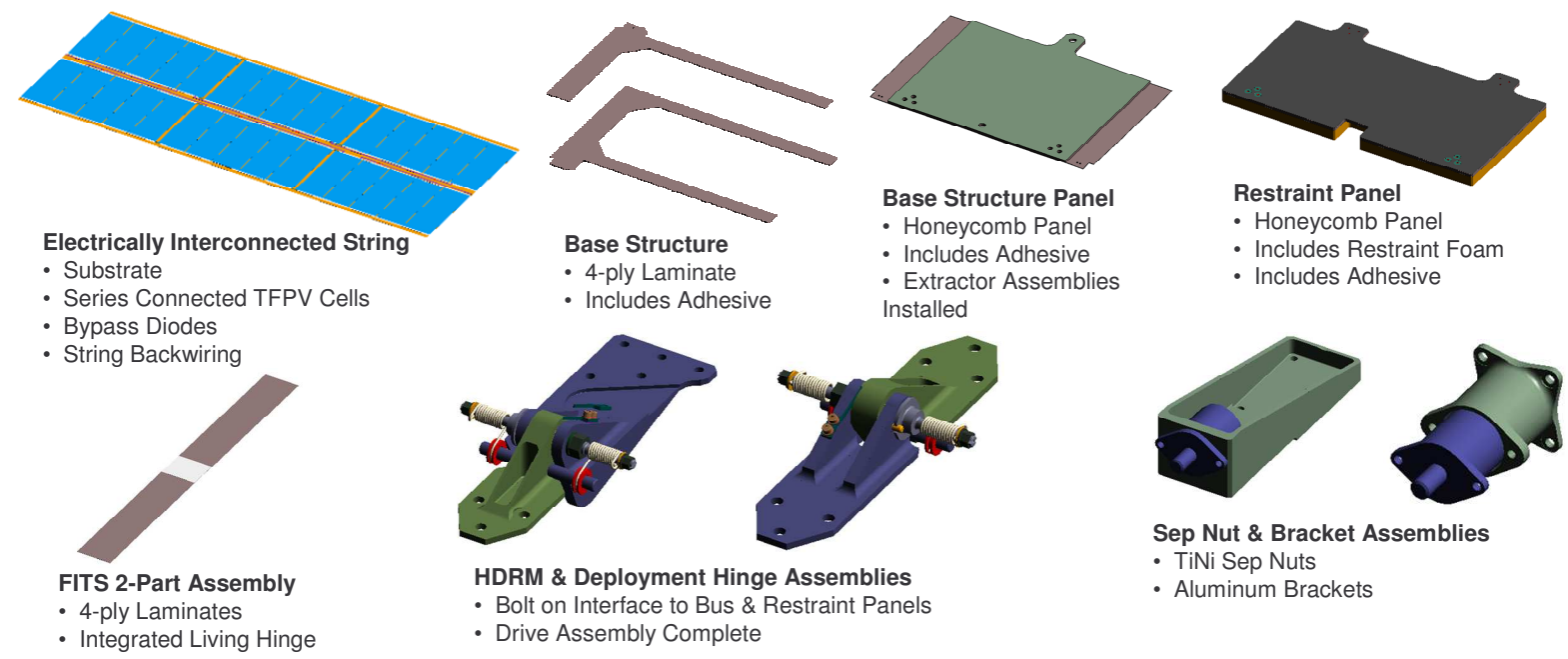

Figure 20. FITS Components Stored In Depot To Support ORS Missions
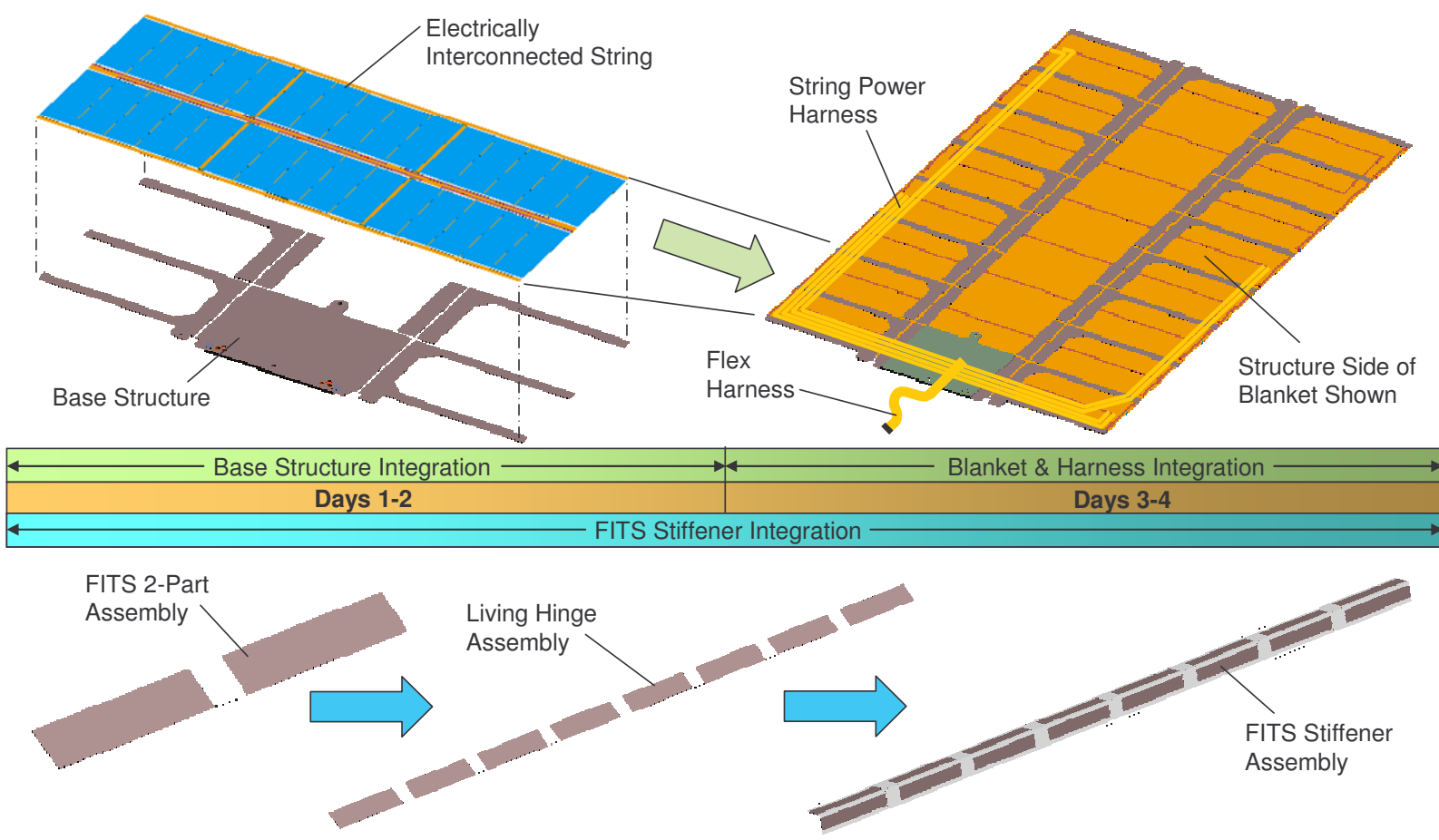

Figure 21. FITS Rapid Manufacturing Timeline, Days 1-4 


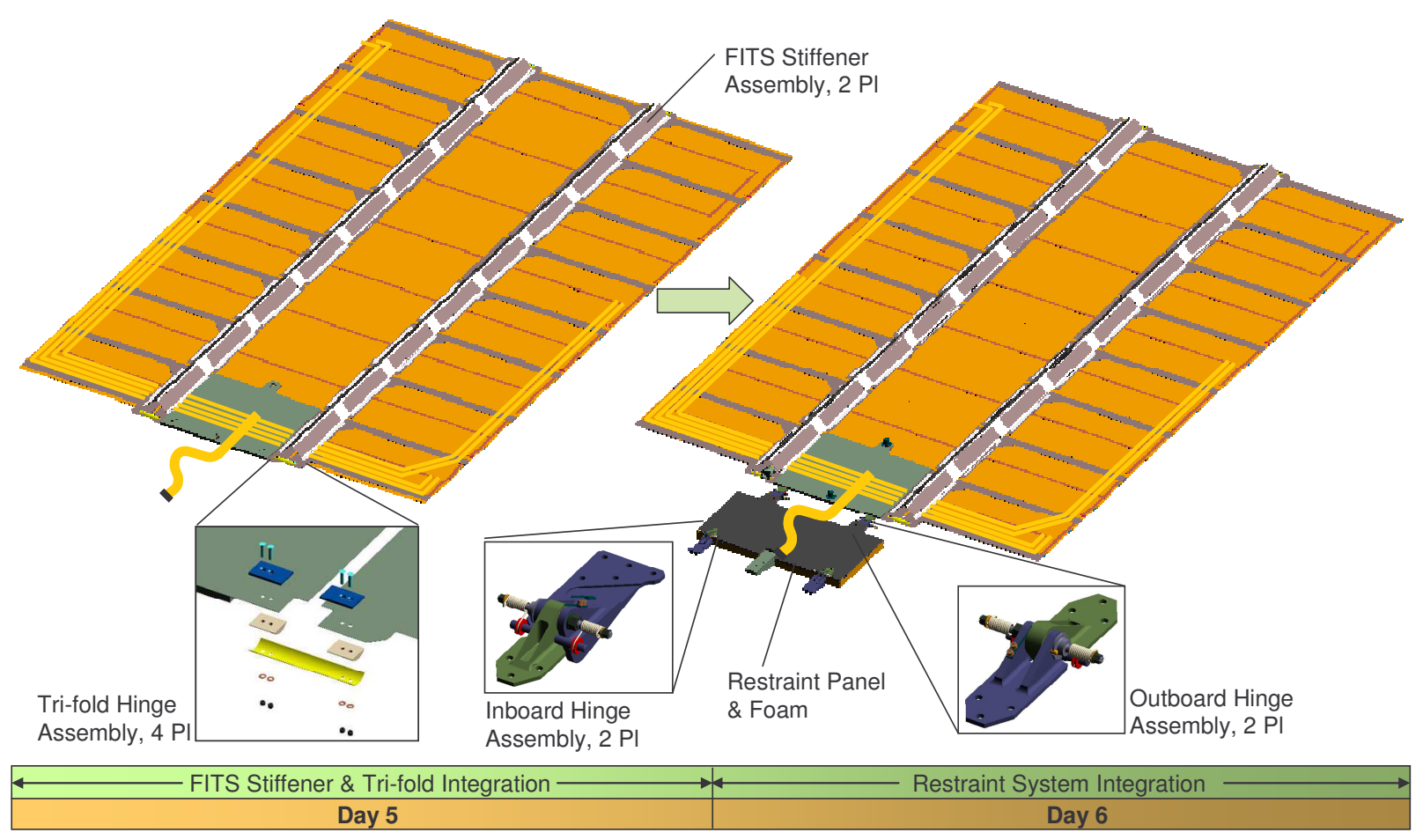

Figure 22. FITS Rapid Manufacturing Timeline, Days 5-6
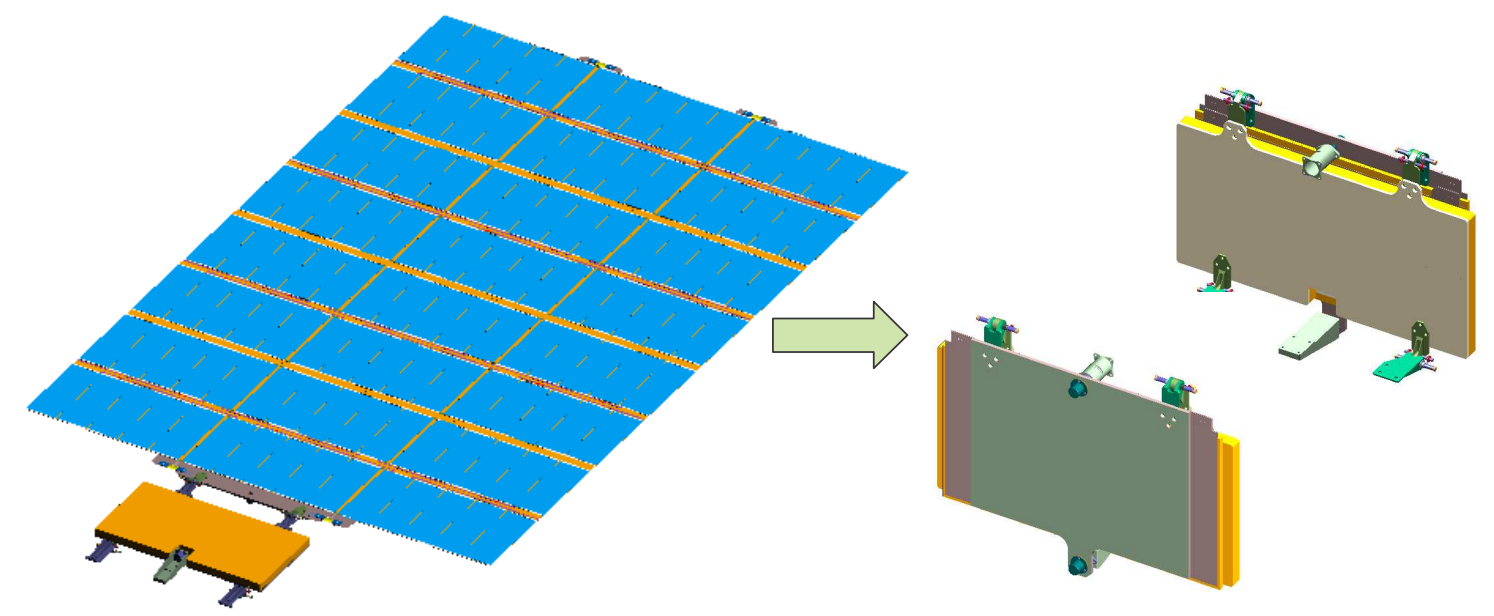

Final Inspection \& Electrical Test

\section{Day 7}

Figure 23. FITS Rapid Manufacturing Timeline, Day 7 


\section{CONCLUSION}

The FITS solar array system developed by MSI is an innovative, low cost, lightweight, solar array system using TFPV that meets power generation needs for future ORS missions. The FITS technology extends the boundaries of space PV systems by eliminating conventional rigid structures and mechanisms and maximizing the lightweight and low stowage volume advantages of TFPV. This innovative approach significantly addresses the schedule and cost constraints of traditional arrays as well as provides significant performance benefits to the customer. MSI has completed the build and qualification test program for a two wing experimental solar array for the AFRL TacSat-2 mission scheduled for launch in November of 2006 and has developed and validated dynamic models of this deployment that can also be used to validate larger scale FITS arrays. The FITS experimental solar array will provide $120 \mathrm{~W}$ of additional power to the spacecraft on top of the primary solar arrays while providing valuable on-orbit performance data of the TFPV to the community for future mission planning. Currently, MSI is under contract from AFRL to design, fabricate, and test a $380 \mathrm{~W}$ EOL FITS wing while focusing on the scalability and modularity of the FITS design. The FITS wing will consist of four modular strings utilizing United Solar Ovonic (USOC) a-Si TFPV material on polymer substrate of approximately $95 \mathrm{~W}$ EOL each resulting in a 2 wing solar array design of approximately $760 \mathrm{~W}$ EOL. MSI has currently completed the design phase of the $380 \mathrm{~W}$ EOL FITS solar array wing and is currently in fabrication. Upon completion of the build, MSI will complete a stringent qualification test program that will validate the FITS solar array for use as a primary power system on small spacecraft.

\section{ACKNOWLEDGEMENTS}

This research is sponsored by the Air Force Research Laboratory, Space Vehicles Directorate. The authors would like to acknowledge the project team at $\mathrm{CU}$ Boulder Center for Aerospace Structures, specifically Dr. Jason Hinkle and Joni Jorgensen.

\section{REFERENCES}

${ }^{1}$ Hinkle, Jorgensen, Zuckermandel, "Ground Verification of a Multi-Stage Deployment Model," AIAA Journal of Spacecraft and Rockets, May 2006. 